\title{
Mouvement maya et culture nationale au Guatemala
}

Shelton H. Davis

Traducteur : Alain Breton

\section{OpenEdition Journals}

Édition électronique

URL : https://journals.openedition.org/jsa/1724

DOI : $10.4000 /$ jsa. 1724

ISSN : 1957-7842

Éditeur

Société des américanistes

Édition imprimée

Date de publication : 5 juin 2004

Pagination : 137-166

ISSN : 0037-9174

Référence électronique

Shelton H. Davis, " Mouvement maya et culture nationale au Guatemala », Journal de la Société des américanistes [En ligne], 90-2 | 2004, mis en ligne le 11 septembre 2014, consulté le 03 septembre 2022. URL : http://journals.openedition.org/jsa/1724 ; DOI : https://doi.org/10.4000/jsa.1724 


\section{II - GUATEMALA}

\section{MOUVEMENT MAYA ET CULTURE NATIONALE AU GUATEMALA *}

\section{Introduction}

En 1981, l'anthropologue mexicain Guillermo Bonfil Batalla publiait une collection de textes écrits par plusieurs jeunes leaders d'organisations indigènes qui mettaient en avant l'émergence d'une nouvelle forme de conscience ethnique parmi les quelque 40 millions d'indigènes d'Amérique latine. Durant la précédente décade, ces leaders et organisations avaient commencé à contester la vision conventionnelle selon laquelle la région était composée d'États-nations monolithiques qui allaient assimiler ou intégrer, socialement et culturellement, leurs importantes populations indigènes. Dans toute l'Amérique latine, des intellectuels d'origine indigène et des leaders des organisations indigènes naissantes mettaient en doute les interprétations généralement acceptées concernant les racines historiques et culturelles de leurs pays, affirmant leurs droits sur les terres et les cultures ancestrales, et appelant à une reconnaissance formelle de la nature multi-ethnique, pluriculturelle et multilingue des sociétés latino-américaines. Bonfil Batalla faisait valoir l'importance des points de vue de ces intellectuels et mouvements indigènes pour le futur des pays d'Amérique latine et il avançait que les instances politiques seraient bien avisées de prendre en compte leurs revendications en termes de reconnaissance officielle, de droits de l'homme et de participation en tant que peuples distincts possédant leur propre identité et des cultures spécifiques ${ }^{1}$.

Depuis la publication du livre de Bonfil Batalla, nombreuses sont ses prédictions concernant l'émergence de nouveaux mouvements indigènes en Amérique latine qui sont devenues réalités. En effet, l'un des résultats de cette émergence a été la transformation des politiques culturelles nationales et la reconnaissance de la diversité ethnique et du pluralisme culturel de la part de nombreux pays latino-américains. Durant les années 1980 et 1990, nombre de ces pays ont réinstauré des régimes démocratiques et libéralisé leur politique économique, ouvrant ainsi de nouveaux espaces politiques correspondant aux revendications des peuples indigènes. La nature de ces changements a pris différentes formes, depuis la réécriture des constitutions nationales et la ratification de la Convention 169 de l'OIT (Organisation internationale du travail) portant sur les Peuples 
indigènes et tribaux, jusqu'à la mise en place de programmes nationaux d'éducation bilingue et interculturelle et la création d'un Fonds hémisphérique pour les Peuples indigènes. Dans des pays comme l'Argentine, la Bolivie, le Brésil, la Colombie, l'Équateur, le Paraguay, le Pérou et le Venezuela, ces changements sont intervenus suite à des protestations démocratiques et à l'action de lobbying sur les gouvernements et les parlementaires exercée par les organisations indigènes. Dans d'autres pays, comme le Nicaragua et le Guatemala - ainsi que le Mexique, suite à la rébellion zapatiste du Chiapas -, c'est par des conflits armés que les droits des indigènes se sont imposés à l'attention nationale et ont été éventuellement reconnus dans le contexte de négociations de paix entre gouvernements et groupes insurgés ${ }^{2}$.

Le présent essai s'intéresse aux origines historiques et sociales de l'un de ces mouvements indigènes, le mouvement maya au Guatemala, et analyse quelquesunes de ses implications pour la culture nationale guatémaltèque, la réforme légale et les politiques publiques. Bien qu'il possède ses particularités et ne soit pas aussi unifié au niveau national que peuvent l'être les mouvements indigènes de Bolivie ou d'Équateur, il a eu un impact important à la fois dans le processus de paix des années 1990 et, plus récemment, dans les politiques nationales concernant l'éducation et la culture, ainsi que dans la discussion préliminaire à la préparation d'une stratégie nationale de réduction de la pauvreté. Plus important, le mouvement maya a connu un relatif succès en acquérant une forte visibilité publique et en obtenant que le Guatemala soit enfin reconnu formellement comme multi-ethnique, pluriculturel et multilingue ${ }^{3}$.

En guise d'introduction, il est important de noter que le Guatemala, contrairement aux autres pays d'Amérique centrale, abrite une population indigène très importante. Selon les sources ou les recensements divers, et selon les critères d'identification retenus, entre 40 et $60 \%$ de la population nationale, estimée à quelque 11 millions de personnes, pratique encore une langue indigène (parmi les 23 langues indigènes reconnues, dont 21 langues mayas et 2 non-mayas, le xinca et le garifuna) et/ou s'identifie comme indigène. Des peuples indigènes, qui résident pour la plupart dans les hautes terres occidentales et les régions septentrionales du pays, qui maintiennent des liens sociaux et historiques forts au sein de leurs communautés, partagent des pratiques traditionnelles d'agriculture et d'usage des terres, ainsi que des traditions artistiques et artisanales mondialement reconnues, notamment à travers les magnifiques textiles produits par les femmes mayas ${ }^{4}$.

Durant les dernières décennies, des transformations majeures ont affecté l'économie rurale du Guatemala, dues en grande partie à la croissance démographique, à la fragmentation et la rareté croissante des terres, et à la migration urbaine et internationale. Dans le même temps, l'extrême pauvreté et le manque d'accès aux services de base se sont accrus massivement. Plus de la moitié de la population nationale du Guatemala $(56,7 \%)$ et près des trois quarts de la population indigène rurale $(73,8 \%$, comparés aux $40,6 \%$ de la population non 
indigène) vivent en dessous du seuil de pauvreté. Au regard de presque tous les indicateurs socio-économiques - revenus et consommation des foyers, éducation et scolarité, santé et nutrition infantile, accès à l'eau potable et conditions d'habitat, etc. -, la population rurale indigène du Guatemala figure parmi les plus pauvres d'Amérique latine et du monde ${ }^{5}$.

S'ajoutent à cela l'exclusion sociale et la discrimination culturelle que la société guatémaltèque a héritées de son passé colonial et post-colonial. Jusqu'en 1945, la législation du travail et des terres était, vis-à-vis de la population indigène majoritaire dans le pays, des plus discriminatoires. Durant la majeure partie de son histoire nationale, l'économie agraire du Guatemala a été caractérisée par une sorte de dualisme structurel dans lequel les terres et la main d'œuvre indigène étaient exploitées aux seules fins du développement agricole capitaliste - c'est-àdire, un système de plantations, à grande échelle, notamment pour la production du café vers les marchés d'exportation ${ }^{6}$.

Dans le même temps, l'idéologie politique et sociale dominante, en particulier depuis la fin du $\mathrm{XIX}^{\mathrm{e}}$ siècle, était celle d'un "nationalisme exclusif ». Cette idéologie, portée par les textes légaux et les écrits des plus éminents intellectuels guatémaltèques, ne reconnaissait ni ne respectait la diversité culturelle du pays. Privés d'accès à l'éducation dans leurs propres langues et privés du droit de vote s'ils ne parlaient pas l'espagnol, les Mayas se voyaient exclus de tout droit «citoyen » formel, et ce jusqu'à la seconde moitié du $\mathrm{xx}^{\mathrm{c}}$ siècle. Le pouvoir politique national et l'idéologie sociale étaient dominés par une petite élite ladina - terme utilisé pour désigner la population non indigène du Guatemala, de culture et de langue espagnole, laquelle connaissait une grande diversité de classes et d'origines - qui montrait peu de respect pour les langues, les cultures, les croyances ou les savoirs de la population maya ?

Cet article retrace les antécédents historiques et sociologiques de la réponse qu'apporte le mouvement maya à ce « nationalisme exclusif » et montre comment ce mouvement a construit un nouveau discours autour de l'idée d'une nation guatémaltèque multi-ethnique, pluriculturelle et multilingue. On commencera par une brève présentation historique de l'apogée de la production du café au Guatemala, de ses effets sur les terres et l'exploitation de la population maya, et de sa relation avec la construction sociale et idéologique de la nation guatémaltèque. Puis, dans un deuxième temps, on présentera quelques-uns des changements les plus importants qui ont affecté la population maya après la Seconde Guerre mondiale, sur le plan social, économique et politique. On s'arrêtera en particulier sur les conséquences de la période dite de la « Révolution nationale », inaugurée en octobre 1944 et interrompue brutalement par le coup d'État, appuyé par les États-Unis, qui renversa le gouvernement du président Jacobo Årbenz Guzmán en juin 1954. Aux lendemains de ce coup d'État, un autre processus, conduit cette fois par les missionnaires étrangers de l'Église catholique romaine et que l'on pourrait qualifier de "réveil sociologique », prit place au sein des communautés 
indiennes. En même temps, la paysannerie indigène se voyait entraînée dans un engrenage généralisé de paupérisation provoqué par l'accroissement démographique et la rareté croissante des terres. En se combinant, la militarisation de l'État guatémaltèque, le réveil sociologique, la mobilisation de la population indigène et la pauvreté croissante alliée à la détérioration de l'économie paysanne rurale vont jeter les bases de la violence politique et de la guerre civile qui déchireront le Guatemala dans les années 1970 et 1980.

Enfin, dans un troisième temps, nous nous focaliserons sur le mouvement maya, les pressions qu'il a exercées pour la ratification de la Convention 169 de l'OIT et son impact sur le projet et, finalement, la signature des Accords de paix en 1996. Concernant ces derniers, l'avancée majeure réside sans aucun doute dans l'accord relatif à l'identité et aux droits des peuples indigènes signé par les représentants du gouvernement guatémaltèque et l'Union révolutionnaire nationale (URNG, organisation regroupant les différents groupes de guérilla) à Mexico, en mars 1995. Bien qu'il y ait eu de nombreuses déconvenues dans l'accomplissement et l'exécution des Accords de paix - y compris le referendum de mai 1999 qui répondit négativement aux nécessaires amendements de la Constitution nationale -, le mouvement maya a joué un rôle majeur en promouvant une nouvelle conception de la nation guatémaltèque fondée sur l'idée de multi-ethnicité, pluriculturalité et multilinguisme. C'est en nous centrant sur ce dernier thème que nous verrons comment cette nouvelle vision de la nation a influé sur les récentes politiques éducatives et culturelles et a pesé de tout son poids dans les débats précédant la mise en place de la stratégie nationale de réduction de la pauvreté. Un argument majeur a été ici que le mouvement maya, malgré ses divisions internes et ses diffïcultés à susciter des réformes constitutionnelles fondamentales (comme ce fut le cas dans d'autres pays d'Amérique latine), a eu des effets significatifs sur la culture nationale du Guatemala. Pour comprendre l'importance de ces changements, il est d'abord nécessaire de remonter dans le temps et de retracer les effets du développement de la production du café sur la population indigène.

\section{La "République caféière » et la création de la nation guatémaltèque}

La plupart des historiens sont d'accord sur le fait que le développement de la production caféière dans le Guatemala de la seconde moitié du XIX ${ }^{\mathrm{e}}$ siècle a eu un impact profond sur l'économie nationale et la population indigène du pays. Ainsi que le souligne le sociologue guatémaltèque, Edelberto Torres Rivas, dans son étude classique sur l'économie et l'histoire sociale de l'Amérique centrale moderne : "l'insertion définitive [de l'Amérique centrale] dans l'économie de marché mondiale » remonte à la fin du XIX ${ }^{\mathrm{e}}$ siècle et à la production et l'exportation du café à grande échelle. Au milieu des années 1880, le café représentait $73 \%$ de la valeur des exportations au Costa Rica, $85 \%$ au Guatemala et $53 \%$ au 
Salvador. Toutefois, on relevait des différences importantes quant aux effets spécifiques que la production du café provoquait sur les structures sociales de ces trois pays d'Amérique centrale. Au Costa Rica, où la plante a été introduite dès le milieu du XIX ${ }^{\mathrm{e}}$ siècle, le café était produit sur de petites exploitations appartenant à des immigrants et reposait sur le salariat d'une main d'œuvre non indienne. En revanche, au Guatemala et au Salvador, l'expansion de la production de café dépendait essentiellement de l'expropriation de terres communales indiennes et de la mobilisation forcée de la main d'œuvre indienne ${ }^{8}$.

À la fin du xix ${ }^{\mathrm{e}}$ siècle, le Guatemala, qui a une population de quelque 1,5 millions d'habitants dont $64,7 \%$ appartiennent à la catégorie « Indiens ", entreprend de préparer le terrain légal pour asseoir la production à grande échelle du café. Dans les années 1870, le président Justo Rufino Barrios, architecte de la Réforme libérale en cette fin du $\mathrm{XIX}^{\mathrm{e}}$ siècle, promulgue une série de décrets permettant d'encourager le développement de la production caféière en Alta Verapaz et dans les régions du piémont de la côte pacifique. Entre 1871 et 1883, le gouvernement de Barrios revend quelque 370000 hectares de «terres publiques », situées dans les zones aujourd'hui les plus riches et les plus productives du pays, à des planteurs de café. La plupart de ces terrains, situés dans les basses terres, telle que la fameuse Costa Cuca qui domine la plaine pacifique, étaient jusqu'alors exploités par des paysans mayas des hautes terres qui y pratiquaient une agriculture de subsistance ${ }^{9}$.

Barrios réinstaure également l'institution coloniale du mandamiento qui confère aux autorités politiques régionales le pouvoir de recruter par la force un certain nombre de personnes au sein de chaque communauté indienne des hautes terres pour les contraindre à travailler pour les nouveaux planteurs de café. En 1877, une loi spéciale concernant la main d'œuvre rurale oblige les travailleurs ruraux à porter et présenter un document sur lequel figurent les obligations de leur contrat de travail, légalise le système des mozos colonos - c'est-à-dire des ouvriers agricoles résidents et des métayers - sur les plantations de café et régule le flux des travailleurs indiens des hautes terres vers les exploitations de café de la côte. Dans le même temps, le gouvernement national encourage l'enregistrement de toutes les terres municipales et communales et l'émission de titres de propriété. Entre 1896 et 1918, pour les sept départements où la concentration indienne était la plus forte, plus de $45 \%$ des lots de terres qui furent concédés par le gouvernement à des particuliers bénéficièrent à des individus de souche espagnole ou européenne. Ces "étrangers » - des ladinos ou des immigrés européens récents - s'étaient installés dans les départements des hautes terres au tournant du $\mathrm{Xx}^{\mathrm{e}}$ siècle, eux-mêmes attirés par le récent boom du café et qui en tiraient profit comme commerçants, vendeurs de liqueur et pourvoyeurs de main d'œuvre ${ }^{10}$.

Du point de vue culturel, deux choses sont à retenir concernant cette période de croissante incorporation du Guatemala et de sa population indigène dans l'économie mondiale du café (et d'autres productions agricoles). La première est 
l'émergence d'une idéologie en vogue au sein de l'élite politique et sociale guatémaltèque (composée de nombreuses personnes d'origine espagnole ou européenne récemment immigrées) qui considérait que la population indigène et ses cultures étaient un obstacle au progrès national. L'une de ses figures les plus marquantes était un avocat guatémaltèque, écrivain et diplomate de surcroît, Antonio Batres Jaúregui. En 1892, à l'occasion du quatrième centenaire de la découverte de l'Amérique, Batres Jaúregui remporta un prix pour son essai sur l'histoire des Indiens mayas. Dans ce texte, il relevait que, bien que la population indienne du Guatemala eût triplé en l'espace d'un siècle, elle manquait de tout dynamisme économique au regard du secteur de la population non indienne. Citant les Principes de l'économie politique de John Stuart Mill, Batres Jaúregui argumentait que les lois relatives au travail forcé réintroduites sous le régime libéral de Justo Rufino Barrios maintenaient la population indienne dans des conditions virtuelles d'esclavage et interdisaient à ses membres toute expérience de la liberté individuelle. Ces lois, d'après lui, expliquaient le niveau extrêmement bas de productivité dans le secteur de l'économie agricole indienne et le fait que le pays était incapable de subvenir à ses besoins en termes d'approvisionnement alimentaire et de production de grains ${ }^{11}$.

Dans cet essai, Batres Jaúregui s'en prend également au système indigène de la propriété communale et à l'inefficacité des lois espagnoles qui reconnaissaient un tel système de tenure foncière. Il dénonçait également le fait que les nouvelles dispositions légales instituées par la Réforme libérale de 1871 pour promouvoir l'agriculture commerciale ne bénéficiaient qu'aux grands propriétaires et au secteur ladino de la population. Les Indiens, écrit-il, « [continuent] de vivre sous un système d'usufruit primitif de propriétés communales, qui relève d'une époque de misère et d'arriération ${ }^{12}$.

Après avoir fait état du retard généralisé de plusieurs villages indiens, Batres Jaúregui suggère au gouvernement de suivre les exemples de l'Argentine ou du Chili, en promulguant un nouveau Code rural qui intègrerait les Indiens dans une économie politique plus large. Un tel code, ajoute-t-il, abolirait les terres communales indiennes, régulerait les besoins en main d'œuvre rurale et préparerait les Indiens à devenir membres d'une société moderne à travers l'apprentissage de l'espagnol et l'introduction de nouvelles aptitudes agricoles et industrielles. « Il est nécessaire, poursuit-il, que les lois agraires, en accord avec l'encouragement au développement de l'agriculture, mène les aborigènes sur la voie de la civilisation " ${ }^{13}$. "Nous devons donner aux Indiens les moyens d'abandonner leur système communal, leurs éternels costumes traditionnels, leur diète barbare faite de totopoxte (grande galette de maïs) et de piment, leurs langues antédiluviennes, leurs maisons primitives, rurales et rustiques. En un mot, les Indiens doivent être extirpés de leur manière d'être - immuable et orientale. Il n'y a aucun doute, alors, que les Indiens seront capables de développer leur civilisation et de participer au progrès ${ }^{14}$. 
Les vues de Batres Jaúregui, ainsi que celles de nombreux autres leaders politiques et intellectuels guatémaltèques en cette période charnière entre $\mathrm{XIX}^{\mathrm{e}}$ et $\mathrm{xx}^{\mathrm{e}}$ siècles, conduisirent à une série de lois encourageant les titres privatifs et la distribution des derniers restes de terres communales indigènes. Elles encouragèrent également l'éducation de la population rurale, l'apprentissage de l'espagnol et sa conversion aux lois, aux habitudes de travail, à la culture du commerce du monde occidental. La théorie dominante concernant l'éducation durant cette époque - bien que rarement mise en pratique si ce n'est dans les grands centres urbains - était fondée sur la notion d'une nation homogène, enracinée dans la culture de l'Europe de l'Ouest et l'abolition des langues et cultures autochtones. Elle était également fondée sur une idée discriminatoire de la citoyenneté qui, depuis la première constitution libérale de 1879 jusqu'à la promulgation de la nouvelle constitution démocratique de 1945, prévoyait que seuls les hommes qui étaient instruits en espagnol avaient le droit de voter et d'occuper des postes politiques à l'échelle nationale ${ }^{15}$.

Le second point qui mérite d'être souligné à propos de cette période de l'histoire du Guatemala est que les Indiens ne réagirent pas passivement face au développement de la production de café comme aux tentatives visant à nier idéologiquement la valeur de leurs langues, cultures et traditions. Ainsi, de nombreux anthropologues qui menèrent leurs recherches au Guatemala dans les années 1920 et 1930 ont pu remarquer les effets profonds que le système des plantations de café produisait sur les peuples mayas. Ces mêmes scientifiques découvrirent également que les Mayas contemporains étaient fermement attachés à leur culture communautaire et possédaient un sens profond de l'identité ethnique locale. Chaque communauté maya des hautes terres possédait un style unique de vêtement et une façon spécifique de parler, pratiquait une endogamie villageoise et maintenait une vie cérémonielle riche rythmée par les anciens calendriers mayas et le culte communautaire des saints catholiques. En d'autres termes, la culture et l'identité mayas avaient résisté, au niveau local tout au moins, aux fortes pressions de l'acculturation qu'exerçaient sur elles le système de la plantation et la société nationale ${ }^{16}$.

En 1941, l'anthropologue Sol Tax, de l'université de Chicago, à l'issue de plusieurs années de travail de terrain dans les villages indiens entourant le lac Atitlán, tenta d'expliquer les raisons pour lesquelles les identités ethniques se maintenaient de façon relativement stable et pourquoi la soi-disant " ladinisation » de la population indienne du Guatemala était si peu marquée. Tax observait que le faible taux d'acculturation des Indiens Mayas ne pouvait être expliqué comme résultant simplement d'obstacles sociaux qui entravaient toute tentative de passage d'une classe (indigène) à l'autre (ladino), ni même d'un manque de proximité physique entre populations indigènes et ladinas. En effet, bien que les ladinos représentassent alors moins de $10 \%$ de la population dans la plupart des villages mayas des hautes terres, ils étaient présents dans 
chacun de ces derniers qui étaient autant de centres sociaux, religieux et commerciaux indiens, et qu'ils entretenaient donc pratiquement des contacts interpersonnels constants avec la population indienne. Or, malgré ces contacts intenses et quotidiens entre groupes ethniques, il y avait très peu de passage d'une classe à l'autre, démontrant s'il en était besoin, selon Tax, qu'il était tout à fait possible « de vivre en contact physique continu avec la périphérie de la civilisation urbaine moderne » et de maintenir cependant une conscience forte de l'identité ethnique et une culture communautaire solidement ancrée dans la tradition et la religion ${ }^{17}$.

Bien que Sol Tax et ses contemporains n'aient jamais caractérisé le phénomène en ces termes, on peut soutenir que, au niveau communautaire tout au moins, un mouvement de résistance ethnique et de revitalisation apparut chez les Mayas du Guatemala durant cette période que les historiens appellent $E l$ siglo del café, « Le siècle du café ». Les travaux anthropologiques ont montré que, durant cette période, les peuples indigènes n'ont pas nécessairement disparu au contact de ce que nous appelons aujourd'hui le "système monde moderne ». Bien au contraire, ces études montrent que les peuples indigènes ont plutôt eu tendance à réaflirmer leur identité ethnique native et leurs traditions culturelles comme un mécanisme de défense face à la perte significative de grandes surfaces de terres communales et à la transformation d'un grand nombre de personnes en un prolétariat rural saisonnier au service des grandes exploitations agricoles capitalistes ${ }^{18}$.

Dans ce processus d'affirmation ethnique à l'époque du formidable développement de l'agro-exportation et de la réforme libérale, trois aspects importants sont à retenir. Premièrement, comme Tax l'avait remarqué, l'identité ethnique traditionnelle était alors essentiellement fondée sur le local et centrée sur l'unité villageoise ou communale (municipio), plutôt que sur des groupes linguistiques ou ethniques plus étendus. Deuxièmement, le mode principal d'organisation servant de canal à l'expression de cette ethnicité était la participation au système des charges au sein des confréries de culte locales (cofradias) ou d'autres organisations dédiées au culte des divinités mayas et des saints catholiques. Troisièmement, l'idéologie sociale de l'identité ethnique indienne traditionnelle tendait à conforter, plutôt qu'à la défier, la division ethnique du travail - Indiens majoritairement paysans et ouvriers agricoles versus grands propriétaires ladinos - sur laquelle était fondée l'économie nationale. Comme nous allons le voir, de nouvelles conditions sociales et économiques naquirent de la Révolution nationale d'octobre 1944 qui ont, à leur tour, mené à une reformulation à la fois de l'idéologie sociale nationale et de l'identité ethnique maya. Cependant, de nombreux aspects de la structure sociale et de la culture guatémaltèques issus de cette période de réforme libérale allaient constituer un bien lourd héritage. 


\section{Le "réveil sociologique » et l'appauvrissement de la population indienne}

Le 20 octobre 1944, un groupe de jeunes officiers et d'étudiants dépose le général Federico Ponce, le successeur désigné du dictateur Jorge Ubico (19311944), et inaugure ce qui allait devenir une ère nouvelle, quoique brève, de gouvernement démocratique et de réformes sociales au Guatemala. Dès les premiers jours du gouvernement du docteur Juan José Arévalo (1945-1951), un ancien instituteur et premier président démocratiquement élu après la Révolution d'octobre, le nouveau gouvernement centre son attention sur les conditions sociales et économiques des secteurs indigènes et ruraux pauvres de la population du pays. La réflexion menant au projet de constitution politique de 1945, par exemple, avait ouvert un débat animé sur les politiques les plus appropriées pour incorporer les communautés indiennes au nouveau projet national de réformes sociales. Entre autres choses, la Constitution de 1945 déclara hors-la-loi toutes les formes de discrimination (Article 21), émit des recommandations afin que les conditions d'hébergement, de santé et de travail pour les " travailleurs indigènes » soient correctes (Art. 67), affirma la nécessité pour le gouvernement de formuler une politique générale pour un plus grand bénéfice social, économique et culturel des groupes indigènes (Art. 83), reconnut que les industries et arts populaires sont des éléments essentiels de la culture nationale (Art. 87) et se prononça pour la pleine reconnaissance et protection gouvernementale de ce qui restait des terres communales (Art. 96) ${ }^{19}$.

S'appuyant sur la Constitution de 1945, le gouvernement introduisit d'autres lois et programmes qui entendaient améliorer sensiblement les conditions socioéconomiques de la population indigène et l'incorporer davantage dans les politiques nationales. Parmi ces nouvelles dispositions : un nouveau code municipal qui prévoyait des élections locales démocratiques, une loi donnant le droit d'affermage des terres aux locataires ou aux occupants, une loi de sécurité sociale et d'autres lois destinées, entre autres, à protéger les textiles indigènes et reconnaître la spécificité orthographique pour l'écriture des quatre langues mayas les plus parlées - K'iche', Kaqchikel, Mam et Q'eqchi'. Le ministère de l'Éducation mit en route également un programme national d'instruction publique (le premier dans l'histoire du pays) et le gouvernement créa l'Institut national indigéniste (INI). Ce dernier avait pour tâche de focaliser son attention sur « les problèmes ethniques auxquels était confrontée la société nationale » et de mettre au point les méthodes les plus appropriées pour « incorporer l'Indien au sein de la culture nationale $\gg{ }^{20}$.

L'INI, dont la création s'inscrivait dans un vaste mouvement tendant à promouvoir l'intégration des peuples indigènes dans les tentatives de réforme sociale, joua un rôle particulièrement important pour dresser l'état des lieux des communautés mayas et décrire les conditions sociales, économiques et culturelles qui étaient les leurs à cette époque. L'une des initiatives les plus marquantes de 
l'institut fut de mener une série d'études de communautés (community studies), en utilisant des techniques anthropologiques modernes (dont la pratique du terrain), qui permit de produire des expertises ethnographiques et socioéconomiques dans plus de 100 communautés mayas. Les résultats montrèrent qu'il existait une grande variabilité dans le degré de traditionalisme ou d'acculturation parmi (et à l'intérieur de) ces communautés indigènes, de même que dans leurs régimes fonciers et leurs systèmes de production ${ }^{21}$.

Dans le cadre de la préparation d'un débat au Congrès autour d'une proposition de loi de réforme agraire, le gouvernement entreprit également le premier recensement agraire national. Le recensement agraire de 1950 confirma le profond dualisme de l'agriculture guatémaltèque, notamment entre l'exploitation à petite échelle du minifindio par la paysannerie indigène des hautes terres occidentales et l'exploitation agro-exportatrice à grande échelle (latifundio) le long de la Côte pacifique. Il souligna également les grandes différences séparant les possessions de terre entre Indiens et ladinos. Par ailleurs, le recensement établit que $72 \%$ des terres de culture du pays étaient contrôlées par $2 \%$ des unités d'exploitation (essentiellement de grandes plantations de café ou de bananes, ou d'élevage de bétail), que $88 \%$ des exploitations (principalement composées de petites unités d'agriculture de subsistance) ne contrôlaient que $14 \%$ des terres, que seuls $10 \%$ des fermes correspondaient à des unités de taille familiale. Alors que de nombreuses petites unités étaient à peine capables de subvenir aux besoins de leurs exploitants, un nombre important de grandes exploitations possédaient des terres non cultivées - un inventaire du ministère de l'Agriculture avait recensé en 1947 plus d'1,75 millions d'hectares de terres ainsi en friche ${ }^{22}$.

Aussi révélateurs furent les résultats d'une autre série d'enquêtes menées par le Bureau de statistiques qui mirent en évidence la continuelle dépendance dans laquelle se trouvaient les petits exploitants ruraux (mayas pour la plupart) vis-à-vis des terres municipales et communales. Les enquêtes menées en 1951, juste avant que ne soit promulguée la Loi de reforme agraire, qui s'appuyaient sur des entretiens auprès d'autorités municipales locales, dévoilèrent que, dans les 22 départements du pays, on comptait encore 505129 hectares de terres municipales et 294458 hectares de terres communales. Les premières correspondaient à quelque 56120 " comuneros » et étaient louées par les corporations municipales à leurs résidents locaux à des fins agricoles, mais aussi pour maintenir des réserves forestières ou servir de pâturages. Les terres communales quant à elles (124 « communautés »), bien que moins étendues que celles détenues par les gouvernements municipaux, correspondaient à un nombre quasi égal de " comuneros » que ceux des terres municipales. Les départements les mieux pourvus de ce type de terres (municipales et communales) se situaient principalement dans les aires d'occupation indigène, soit les hautes terres occidentales et la partie orientale du pays ${ }^{23}$. 
La Loi de réforme agraire (ou Décret 900) fut introduite par le gouvernement nouvellement élu du colonel Jacobo Árbenz Guzmán en 1952 et devint bientôt l'objet d'une des plus vives controverses de son administration. Ainsi que de nombreux commentateurs l'ont souligné, autant le Décret 900 apportait une solution radicale aux problèmes agraires du Guatemala, autant l'un de ses objectifs explicites était d'introduire le « capitalisme agricole » dans les campagnes du Guatemala, un objectif qui - fait intéressant - était partagé par des émissaires de la Banque mondiale qui visitèrent le Guatemala et exposèrent les résultats d'une étude au colonel Árbenz Guzmán trois mois après qu'il ait pris possession de sa charge en juin 1951. Cependant, la Loi de réforme agraire et quelques-uns des plus proches conseillers d'Árbenz prônaient la mobilisation de masse des paysans, des fermiers et des ouvriers agricoles au sein de ligues paysannes, de syndicats et de comités agraires. La loi prévoyait également l'expropriation et l'éventuelle redistribution aux paysans (en tant qu'usufruitiers) des terres qui, possédées privativement, n'étaient pas cultivées. De plus, elle contenait un article (Art. 9) qui établissait que les terres municipales laissées en friche (sauf celles désignées comme "réserves forestières » et placées sous le contrôle et la gestion des instances nationales) pouvaient être dénoncées par les comités agraires ; tandis qu'un autre article (Art. 33) stipulait que ces terres, dès lors qu'elles étaient l'objet de conflit entre les municipalités et les comités agraires, devaient être adjugées à ces derniers ${ }^{24}$.

Il n'est donc pas surprenant, étant donné l'agitation politique et les tensions interethniques qui se manifestaient dans de nombreux villages indiens, que des conflits aient éclaté, notamment entre gouvernements municipaux et comités agraires comme entre ceux-ci et les ligues paysannes récemment formées. La nature de ces conflits dépendait de la composition ethnique et de l'histoire politique de chaque village indien comme de la rapidité avec laquelle les partis politiques étaient en train de conquérir les niveaux municipal et national. La prise de contrôle des terres municipales et leur distribution lors de la Réforme agraire se virent également empêtrées dans des disputes religieuses au niveau local, parce que des sectes protestantes nouvelles commençaient à affirmer une forte présence dans les campagnes et qu'un mouvement réformateur, appelé Action catholique, commençait à compter et à entrer en conflit avec les formes religieuses plus traditionnelles de croyances et de pratiques mayas-catholiques. Ce qui apparaît clairement dans les données d'archives, comme dans les écrits des observateurs de la vie rurale et les témoignages oraux des activistes paysans ayant connu (et survécu à) cette époque, est que la lutte pour la terre devint la source d'intenses conflits politiques, tant au niveau national que des communautés villageoises, lors de la mise en place de la Réforme agraire ${ }^{25}$.

C'est dans cet environnement politique général qu'un autre facteur va bien évidemment nourrir les conflits intra- et intercommunaux et qui se fera d'ailleurs sentir au-delà de ladite période : le facteur démographique. De fait, au Guate- 
mala, à partir de 1950 (date à laquelle on dispose de recensements nationaux modernes), la population montre un taux de croissance très rapide. Le recensement de 1950 dénombrait une population nationale de 2,9 millions d'habitants, et presque chaque décompte depuis cette date indique un taux de croissance annuel compris entre 2 et $3 \%$ à l'échelle nationale, et des taux encore plus élevés dans la population indigène. Les conséquences sociales et écologiques de cette augmentation démographique furent des changements fondamentaux dans l'usage des terres à l'intérieur des communautés rurales et indigènes (entraînant la réduction des temps de jachère et de rotation des cultures), une intensification de la fragmentation des terres, la dépossession totale pour certains et les migrations internes. L'accroissement de la population a également affecté l'intégrité des possessions municipales et communales dès lors que davantage de gens étaient en quête de terres pour subvenir à leurs besoins vitaux et qu'une pression accrue se faisait sentir sur ces terres-là pour répondre aux nouvelles et impérieuses nécessités en pâtures, bois de construction et bois de chauffage ${ }^{26}$.

De fait, au début des années 1950, se dessine un processus de mobilisation politique dans la plupart des communautés mayas du Guatemala. Dans le même temps, résultat de l'accroissement démographique, de la fragmentation et de la raréfaction des terres, on constate une détérioration générale des conditions de vie de la majorité des paysans mayas qui se voient obligés de trouver d'autres moyens d'assurer la subsistance et la survie de leurs familles. Ce sera pour beaucoup la migration interne, comme travailleurs saisonniers vers les plantations de café et celles, plus récentes, de coton sur la Côte pacifique. Le coup d'État militaire de juin 1954 marque la fin brutale de la Réforme agraire et, tout à la fois, de l'essentiel de la mobilisation politique qui agita les régimes d'Arévalo et d'Árbenz. Cependant, l'appauvrissement des populations rurales s'accroissant, de nouvelles formes de mobilisation sociale et politique vont se faire jour dans les années suivantes ${ }^{27}$.

Le facteur le plus important, sans doute, de la poursuite de la mobilisation sociale et politique de la population indigène fut le mouvement d'Action catholique qui, établi au Guatemala en 1948, crût de façon significative après le coup d'État militaire de 1954. À l'origine, le mouvement d'Action catholique était une tentative de la part de la hiérarchie de l'Église pour contenir la marée montante du fondamentalisme protestant et des politiques radicales (dans le contexte de la Guerre froide, fortement " anti-communiste ») qui gagnaient du terrain et de la popularité parmi les populations mayas. En s'amplifiant, le mouvement religieux devint la base d'un formidable mouvement de revitalisation ethnique et de modernisation rurale ${ }^{28}$.

Des sociétés missionnaires catholiques, qui avaient été bannies du Guatemala à l'époque libérale, jouèrent un rôle majeur dans le développement du mouvement d'Action catholique et l'influence sociale et culturelle qu'il allait exercer. Entre 1950 et 1965, par exemple, le nombre de prêtres catholiques au Guatemala 
augmenta de 132 à 483, alors que le nombre de religieuses passa de 96 à 354 . L'essentiel de cette augmentation des personnels religieux fut le fait de congrégations et d'ordres étrangers qui, à l'origine, envoyaient leurs missionnaires pour mener des activités plutôt conventionnelles visant à convertir les tenants de la religion maya traditionnelle (les membres des confréries en priorité) à un catholicisme réformé, fondé sur la participation rituelle à la vie sacramentale de l'Église. Cependant, grâce à la formation de catéchistes indiens ou de leaders laïcs, le clergé commença à transformer les perspectives sociales et politiques des Indiens qui, dans le même temps, prenaient conscience par eux-mêmes de la situation. Ces ordres et congrégations commencèrent également, sous l'influence philosophique du concile Vatican II et du mouvement de la Théologie de la libération qui émergeait en Amérique latine, à canaliser les nouveaux convertis dans les mouvements paysans et coopératifs naissants ; dans quelques régions du pays, ils posèrent les prolégomènes de ce qui allait devenir, dans les années 1970, « l'Église du peuple » ${ }^{29}$.

Au début des années 1970, les partis politiques d'opposition, et notamment le parti de la Démocratie chrétienne (DC), commencent de leur côté à organiser (et gagner de l'influence sur) les campagnes guatémaltèques. Malgré la victoire, frustrée par les militaires, lors des élections présidentielles de 1974, la DC et d'autres partis remportèrent nombre d'élections municipales au sein des communes indiennes ${ }^{30}$. À la suite du tremblement de terre qui dévasta le pays en février 1976, on assiste à un regain d'activité politique de la part du mouvement syndical urbain, qui aura également une influence sur les populations rurales indigènes. Bien qu'il n'ait jamais atteint le degré d'unité et d'organisation qu'il avait connu aux temps des régimes d'Arévalo et d'Árbenz, le mouvement syndical touchait de nouveaux secteurs industriels de la population urbaine, ainsi que des travailleurs agricoles et des paysans. Le fait le plus marquant dans cette revitalisation du mouvement ouvrier au Guatemala fut, probablement, la formation en avril 1976 du Comité national d'union syndicale (CNUS). Dès sa création, le CNUS entretint des liens étroits avec les organisations de travailleurs paysans et ruraux, tout en gardant son indépendance vis-à-vis des partis politiques radicaux et du mouvement naissant de guérilla. En 1978, le CNUS, allié à d'autres organisations religieuses, politiques et ouvrières, commença à protester contre l'accroissement de la présence militaire dans les zones rurales. Des juristes du CNUS assistaient également les coopératives paysannes créées dans les zones frontières qui rencontraient d'importantes difficultés pour obtenir l'enregistrement officiel des terres ; de même, des membres de la fédération syndicale rejoignirent les mineurs en grève dans les régions à dominante indigène comme le Huehuetenango et la Verapaz ${ }^{31}$.

La fin des années 1970 voit aussi la formation du Comité d'union paysanne (CUC) dont l'objectif est de resserrer les liens entre les divers mouvements syndicaux ruraux et urbains et le gros de la paysannerie indigène du pays. Le CUC fut, dans toute l'histoire du Guatemala, la première organisation syndicale 
dirigée par des Indiens et la première à rallier en son sein paysans indigènes des hautes terres et agriculteurs ladinos pauvres. Bien que contraint à travailler dans la clandestinité, il est probable qu'il bénéficiait de l'appui de milliers de travailleurs agricoles, permanents et saisonniers. Sa principale action publique, avant son ralliement au mouvement de guérilla, fut l'organisation en février 1980 d'une grève réunissant quelque 70000 coupeurs de canne à sucre et 40000 cueilleurs de coton, une action qui, avec d'autres du même genre (grève dans les plantations de café en septembre 1980), força le gouvernement à augmenter le salaire minimum des travailleurs agricoles, le faisant passer de 1,12 à 3,20 dollars par jour ${ }^{32}$.

En somme, dès la fin des années 1970 , les premières pierres d'une mobilisation sociale et politique généralisée de la population rurale indigène du Guatemala étaient posées. Bien que la mobilisation fût plus ou moins importante selon les régions et les groupes ethnolinguistiques, voire au sein même des communautés indigènes, on ne peut guère mettre en doute que les aspirations sociales, économiques et politiques de la population maya aient été encouragées et relayées par les activités des missionnaires étrangers, par les agents du développement rural, par les partis politiques d'opposition et par les mouvements syndicaux urbains et ruraux. Sous certaines conditions socio-politiques plus favorables - telles que la démocratie, la réforme sociale et le développement économique global -, une telle mobilisation aurait pu naturellement évoluer vers une plus grande participation des Indiens à la société et à la vie publique nationales. Cependant, compte tenu de la situation réelle, encourager les Indiens à participer en tant qu'acteurs libres et indépendants à la politique nationale était on ne peut plus contraire au projet des militaires. Ce n'était pas davantage dans le projet des mouvements de guérilla, récemment réorganisés (au sein de l'URNG), que d'accepter un changement au Guatemala qui suivrait une voie non violente. Alors, comme toute la région d'Amérique centrale qui s'enfonçait dans un tumulte politique et militaire, le Guatemala entra dans une guerre civile à grande échelle que les Mayas furent les premiers à subir, victimes des déplacements et de la violence. C'est sur les cendres d'un des conflits les plus meurtriers, des campagnes anti-insurrectionnelles parmi les plus brutales qu'ait connu l'histoire moderne de l'Amérique latine, qu'après un retour à la démocratie en 1985, le mouvement maya actuel émergea sur la scène politique du Guatemala ${ }^{33}$.

\section{Le mouvement maya et les Accords de paix}

Tout au long des années 1970 et plus encore dans les années 1980, de jeunes indigènes en nombre croissant commencèrent à redécouvrir l'importance historique de leurs racines culturelles mayas et à réaftirimer leurs identités ethniques. Plusieurs facteurs influèrent dans cette redécouverte et cette aftirmation de la part de cette nouvelle génération de leaders et d'intellectuels mayas. Durant toutes ces 
années, un petit nombre de jeunes Mayas purent accéder à l'éducation secondaire et supérieure et commencèrent à s'interroger sur eux-mêmes et surtout, publiquement, sur les idées dominantes concernant l'histoire et la dynamique de la politique, de la société et de la culture au Guatemala. En particulier, certains de ces jeunes Mayas réagirent fortement face aux théories alors défendues par des anthropologues nord-américains relatives à la supposée "ladinisation 》 de la population maya, comme à celles d'historiens et de sociologues guatémaltèques qui ne voyaient dans les mouvements mobilisant la population indigène qu'un témoignage de l'intensification de la «lutte des classes » dans les zones rurales. Dans le même temps, tandis que ces nouveaux leaders indigènes mettaient en question les interprétations communes de l'histoire et de la société guatémaltèques, un nombre important d'autres jeunes Mayas se focalisaient sur la revalorisation de leur héritage linguistique et culturel au contact de linguistes et d'anthropologues étrangers. Beaucoup d'entre eux furent également influencés par le professeur Adrián I. Chávez, un éducateur maya-k'iche' de Quetzaltenango, qui fut le premier traducteur indigène du Popol Vuh (ou Pop Wuh), le livre sacré des Mayas-K'iche'. De nouvelles organisations et associations vont alors donner corps à ces initiatives qui, au début des années 1990, dessinent un mouvement maya diffus mais de plus en plus présent sur la scène publique, un mouvement qui allait, à son tour, et pour la toute première fois dans l'histoire du Guatemala, exercer une influence significative dans les débats concernant l'avenir du pays ${ }^{34}$.

Trois points concernant la dynamique sociale et l'influence du mouvement maya méritent d'être soulignés. Premièrement, à l'image d'autres mouvements indigènes qui ont surgi à la même époque en Amérique latine, celui-ci a bénéficié de l'attention internationale portée aux droits des peuples indigènes, spécialement dans le contexte de la Commission des droits de l'homme des Nations unies et de l'Organisation internationale du travail (OIT). Plusieurs leaders du mouvement maya ont participé aux réunions et discussions du Groupe de travail sur les populations indigènes des Nations unies (créé en 1982), notamment sur le projet de Déclaration des droits des peuples indigènes, et aux débats qui, dès 1989, ont mené à la ratification par le Guatemala de la Convention 169 de l'OIT. Il y eut également des contacts entre ces leaders mayas et d'autres leaders indigènes d'Amérique lorsqu'il s'est agi de préparer une riposte à la célébration (par l'Espagne et plusieurs autres gouvernements latino-américains) du " Cinquième centenaire de la découverte de l'Amérique » en 1992. Les organisations indigènes substituèrent au titre offíciel des célébrations à venir l'intitulé « Cinq cents ans de résistance 》 et mirent sur pied une grande réunion à l'échelle continentale à Quetzaltenango en 1991. L'annonce de l'attribution du prix Nobel de la paix, en octobre 1992, à Rigoberta Menchú Tum, militante des droits des indigènes au Guatemala, conféra une impulsion décisive au mouvement maya et contribua grandement à sa visibilité internationale ${ }^{35}$. 
Deuxièmement, rappelons que le mouvement maya est très fragmenté et n'est en aucun cas représenté par un seul courant idéologique, ni même par une organisation qui pourrait s'exprimer en son nom. De fait, ainsi que plusieurs observateurs ont pu le constater, il existe souvent de profondes divergences à l'intérieur du mouvement entre ceux qui défendent un point de vue essentiellement « culturel »- et, parmi eux, de nombreux intellectuels et écrivains mayas et ceux - leaders indigènes pour la plupart - qui continuent à revendiquer des liens forts avec certaines des organisations populaires défendant la « lutte des classes ", organisations qui existaient déjà bien avant le conflit. La nature de ces divergences fut mise en avant lors de la seconde réunion continentale pour la résistance indigène, noire et populaire qui se tint à Quetzaltenango en 1991, et que certains intellectuels mayas dénoncèrent comme ayant été manipulée par le « mouvement populaire » et « la Gauche ». D'autres ruptures existent au sein du mouvement maya selon d'autres lignes, incluant les différences de langues, de lieux de résidence (organisations rurales ws. urbaines) et de religions. Concernant ces dernières, on assiste à une extrême fragmentation des croyances et des pratiques religieuses : aux divisions déjà existantes entre les costumbristas (mayascatholiques traditionnels), les catholiques réformés et de multiples groupes évangéliques protestants, vient se surajouter le courant porté par la redécouverte et l'affirmation des coutumes, valeurs et croyances "mayas » de la part de plusieurs leaders indigènes au tournant des années 1980-1990. À plusieurs reprises depuis, et spécialement pendant et après la signature des Accords de paix, des tentatives ont été faites en vue d'unifier les diverses organisations mayas et populaires, mais, contrairement à d'autres pays (Bolivie, Colombie, Équateur...), aucune organisation indigène n'a émergé au Guatemala pour représenter et s'exprimer au nom d'une population indigène nombreuse et très hétérogène ${ }^{36}$.

Troisièmement, alors que le mouvement maya gagnait en visibilité et que ses demandes en termes de droits indigènes et humains imposaient une nouvelle vision de la société et de la culture nationales guatémaltèques, il provoqua un contrecoup parmi les élites politiques, intellectuelles et autres. Le nouveau dessein d'un Guatemala multi-ethnique, pluriculturel et multilingue, ainsi que les revendications portant sur la reconnaissance et la protection des identités et des droits de la population indigène soulevèrent de sérieuses craintes parmi certains secteurs politiquement et socialement dominants de la population ladina qui voyaient dans le mouvement maya une menace portée à l'unité de l'État. Bien que plusieurs leaders du mouvement maya déclarèrent qu'il n'était aucunement question de se séparer de la nation guatémaltèque ou de fomenter une nouvelle forme de "nationalisme exclusif », la peur s'empara d'une grande partie des secteurs non indigènes de la population et alimenta de nombreux débats au sein des cercles politiques et intellectuels, comme dans la presse nationale ${ }^{37}$.

Ces discussions allaient tenir la vedette lors des débats publics concernant la ratification de la Convention 169 de l'OIT et de l'exécution des Accords de paix, 
notamment de l'accord portant sur l'identité et les droits des peuples indigènes. Si la Constitution du Guatemala de 1985 comportait déjà toute une section et plusieurs articles relatifs aux "communautés indigènes ", aucun amendement sérieux n'avait été proposé depuis lors pour anticiper cette évolution. En fait, ce n'est qu'au tournant des années 1990 que le Congrès du Guatemala, saisi de la question de la ratification de la Convention 169, se fit le relais d'un débat public relativement houleux. Ladite convention, proposée par l'OIT en 1989, était en net progrès par rapport à la Convention 107 de 1957, en proposant un cadre qui laissait une plus large place au pluralisme et à la participation pour tout ce qui concernait les populations indigènes. De même, elle contenait plusieurs clauses relatives à la reconnaissance et la protection des terres et des ressources naturelles indigènes, mais aussi des lois coutumières et des procédures légales au sein des communautés indiennes ${ }^{38}$.

Au début des années 1990, une série de discussions prit place au sein des organisations indigènes et de la société civile quant au contenu de la Convention 169 et à son importance pour le Guatemala. Malgré le plein appui du ministère de Travail, mais un soutien nettement plus mitigé de la part du gouvernement et de plusieurs membres éminents du Congrès, les demandes expresses de ratification formulées par les organisations mayas provoquèrent finalement un effet boomerang de rejet de la part de certaines formations, reflétant l'opinion dominante des élites militaires, juridiques et politiques. La polémique autour de la ratification de la Convention 169, qui opposa le mouvement maya au Congrès (où les députés mayas sont largement sous-représentés), tarda presque quatre ans... jusqu'à ce que le Congrès, poussé dans ses derniers retranchements par les recommandations des Accords de paix, daigne finalement, en 1996, ratifier la convention. Avec une réserve de taille toutefois puisqu'une clause stipulait que la Convention 169 devait être subordonnée à la Constitution, une réserve qui laissait malgré tout ouverte la possibilité de débattre et d'interpréter à l'envi les recommandations de la Convention 169 et la façon dont le Guatemala devait traiter sa population indigène ${ }^{39}$.

Les querelles politiques internes mettant aux prises le mouvement maya, le gouvernement et le Congrès national, ainsi que les secteurs les plus puissants de la société nationale guatémaltèque permettent de mieux comprendre les controverses qui suivirent la signature offícielle des Accords de paix en décembre 1996. Comme mentionné plus haut, il est clair que le document principal du point de vue des Mayas était l'Accord sur l'identité et les droits des peuples indigènes (ci-après AIDPI). Bien que cet accord ait été rédigé par le gouvernement et l'URNG, sans participation directe des organisations mayas, ces dernières eurent cependant une influence sur son contenu, ne serait-ce qu'à travers leur représentation au sein de l'Assemblée de la société civile (ASC). L'ASC, créée pour assurer un degré minimum de participation de la société civile guatémaltèque dans le processus de paix, représentait onze secteurs de la société civile, dont la Coordi- 
nation des organisations du peuple maya de Guatemala (COPMAGUA, encore appelée Saqb'ichil Copmagua). Forte d'une approche consensuelle caractéristique de la prise de décision collective telle qu'elle se pratique traditionnellement chez les Mayas, COPMAGUA et ses nombreuses organisations affiliées eurent un impact significatif sur le contenu de l'AIDPI, de même que sur les discussions qui s'ensuivirent (avec le gouvernement, les autres organisations de la société civile et les représentants de la communauté internationale) en vue de son application ${ }^{40}$.

Entre autres choses, cet accord ébauche les contours d'un cadre institutionnel en vue de la création d'une nation multi-ethnique, pluriculturelle et multilingue au Guatemala. Pour cela il appelle de ses vœux la reconnaissance des identités historiques des communautés guatémaltèques de langues mayas, xinca et garifuna, et propose que priorité soit donnée à la lutte contre toutes formes de discrimination sociale, raciale, culturelle et sexuelle. Il promeut également les droits culturels des peuples indigènes, y compris les droits de pratiquer leurs langues, de choisir leurs noms individuels, de cultiver leurs croyances spirituelles, de fréquenter leurs centres religieux et cérémoniels, de préserver leurs lieux sacrés, leurs costumes traditionnels, leurs connaissances, leur science et leurs savoir-faire. Une disposition majeure de l'accord concerne la « réforme de l'éducation » qui, en toute logique au regard des autres dispositions, se doit d'être cohérente avec la pluralité des cultures et des langues du pays. Parmi les mesures proposées figurent un appui gouvernemental plus important au Programme d'éducation bilingue et interculturelle (PRONEBI), une plus grande décentralisation et régionalisation des systèmes d'enseignement afin qu'ils correspondent au plus près à cette réalité plurielle, et des révisions fondamentales des programmes qui prennent pleinement en compte les conceptions indigènes de l'éducation. Une commission spéciale, bipartite, composée de représentants du gouvernement et des organisations mayas fut créée - et fonctionna effectivement de 1996 à 1999 - pour préciser les recommandations concrètes qui permettraient la mise en œuvre d'une telle réforme de l'enseignement ${ }^{41}$.

En termes de droits civils et politiques, et en accord avec les objectifs fixés, l'AIDPI soulignait la nécessité de modifier la Constitution. Il se prononçait également en faveur d'une reconnaissance pleine et entière des communautés indigènes locales et de leurs représentants traditionnels, du rôle actif de ces communautés dans la décentralisation administrative, de la participation effective des peuples indigènes et de leurs organisations à tous les niveaux (local, départemental, national) de gouvernement et d'administration, ainsi que d'une semblable reconnaissance des lois coutumières tant à l'intérieur des communautés qu'à l'échelle des juridictions régionales et nationales ${ }^{42}$.

Enfin, c'est toute la question des droits fonciers que l'AIDPI traite en priorité. Une section spécifique de l'accord - la section IV-F - est consacrée aux droits relatifs aux terres des peuples indigènes : « ces droits, est-il précisé, incluent les tenures communales et collectives autant que les tenures individuelles [de ces 
terres] ; les droits de propriété, d'usufruit et autres régimes, ainsi que les droits liés à l'usage des ressources naturelles au bénéfice des communautés sans préjudice pour leur environnement $\gg{ }^{43}$. Une attention spéciale est portée à la nécessité de protéger juridiquement les droits des communautés indigènes. Ce qui implique le développement de normes légales spéciales sur les terres indigènes, la formation de juges locaux en vue de la résolution des conflits de terre au sein des communautés ou entre elles, une assistance légale dans les cas de réclamations indigènes sur les terres, la mise à disposition d'interprètes bilingues et la circulation de l'information concernant les droits agraires et les procédures légales parmi les populations indigènes. On rappellera qu'il existe un accord séparé, portant sur les aspects socio-économiques et la situation agraire, signé par le gouvernement et l'URNG, mais il souff're de graves lacunes quant à la question des terres indigènes.

Avec des propositions d'une telle ampleur, il n'est pas surprenant que l'AIDPI et les autres accords aient provoqué un séisme politique majeur au Guatemala lorsqu'il s'est agi de leur application. Le principal point d'achoppement fut sans doute celui portant sur la nécessité de réformes constitutionnelles. Pour ne prendre que l'AIDPI, plusieurs propositions fondamentales de réforme auraient dû s'appliquer à la Constitution de 1985 afin de créer les conditions requises pour ce nouveau dessein de nation. Mais lorsque l'ampleur de ces réformes fut connue et prise en considération, une vive polémique opposa les organisations indigènes et celles de la société civile aux énormes intérêts et droits acquis des élites politiques, économiques et militaires du pays. Le gouvernement en place à l'époque de la signature des Accords de paix - sous la présidence d'Álvaro Arzú Irigoyen (1996-2000) -, dans l'incapacité de réunir un consensus des congressistes de son propre parti politique comme des partis d'opposition, se vit obligé de recourir au referendum. Cinquante propositions de réforme constitutionnelle furent initialement soumises au Congrès national ; plusieurs d'entre elles concernaient les terres indiennes, la reconnaissance officielle des langues indigènes et les droits des peuples autochtones à s'administrer selon leurs lois coutumières. De même figurait une proposition pour redéfinir le Guatemala en tant que « nation multi-ethnique, pluriculturelle et multilingue » qui aurait des implications fondamentales dans la structure politique et administrative du pays.

Le referendum sur les réformes proposées eut lieu en mai 1999. Durant les mois qui précédèrent la consultation, la presse et les médias nationaux menèrent de grandes campagnes sur les conséquences des Accords de paix. La résistance la plus forte fut celle du Front républicain guatémaltèque (FRG), parti de l'opposition, et de plusieurs organisations « constitutionalistes » conservatrices telles que le Centre pour la défense de la Constitution (CEDECON), la Ligue de la Mère-Patrie, l'Association des dignitaires de la Nation, et d'autres encore. Le puissant Comité coordinateur des associations agricoles, commerciales, industrielles et financières (CACIF) - équivalent guatémaltèque du MEDEF français $(\mathrm{NdT})$ - s'exprima haut et fort contre les changements proposés arguant que le 
paquet complet des cinquante projets de réformes, tout comme les quatre questions soumises au vote des citoyens (l'une d'elle ayant directement trait aux droits des peuples indigènes), constituait « une violation de la volonté du peuple ». Le referendum eut finalement lieu, mais moins de 18,5\% des électeurs inscrits se rendirent aux urnes et $55 \%$ d'entre eux votèrent contre les réformes constitutionnelles. Ainsi que l'a justement souligné Suzanne Jonas ${ }^{44}$, qui passe pour l'une des meilleures observatrices et analystes du processus de paix au Guatemala, la victoire du non au referendum de mai 1999 renforça un sentiment de triomphe parmi les secteurs qui s'étaient opposés depuis le début à tout processus de paix. Aux États-Unis, un article de presse résumait froidement la situation en titrant "Guatemalans turns thumbs down on multiculturalism » [Les Guatémaltèques tournent le dos au multiculturalisme] (Wall Street Journal, 28 mai 1999).

Bien que l'issue de ce referendum représente un sérieux revers pour le mouvement maya et un déni d'une évolution possible vers une société plus pluraliste, il doit se comprendre aussi historiquement et à la lumière de la politique et de la culture contemporaines du pays. Pendant plus d'un siècle et demi, la population indigène majoritaire du Guatemala - répartie en 23 groupes ethnolinguistiques distincts, des centaines de bourgs et des milliers de petites communautés locales a été systématiquement écartée des droits à la citoyenneté et de la politique nationale. Ce « nationalisme exclusif », caractéristique du pays, est très fortement ancré dans les mentalités; il marque profondément les attitudes culturelles et les relations interethniques, et les systèmes de contraintes politiques et administratives comme, plus récemment (lors de la campagne anti-insurrectionnelle des années 1970-1980), la prégnance du contrôle militaire n'ont fait que le renforcer. Changer cette structure de domination sociale, politique et culturelle, notamment en cherchant à induire un nouveau cadre de débat démocratique et de politique électorale, représentait un immense défi de la part du mouvement maya. La cause était loin d'être gagnée compte tenu de la fragmentation même du mouvement comme des populations qu'il représente face à la puissance du contrôle que les élites traditionnelles continuent de maintenir sur l'appareil politique des partis et sur les media. De là, on peut considérer que le referendum de mai 1999 ne fut pas l'échec d'un projet pour une société culturellement plus pluraliste et ouverte, mais simplement un obstacle de plus sur la voie suivie par le mouvement maya et ses alliés au sein de la société civile, une direction d'ailleurs partagée par certains secteurs du gouvernement (en particulier le Secrétariat à la paix et la Commission pour la paix nationale, établis pour appliquer les Accords de paix) et la communauté internationale. Comme nous le verrons dans la dernière partie, bien que cette vision d'une nouvelle société n'ait pas été confortée par le referendum, elle a influencé un vaste débat public et suscité de nombreuses initiatives dans différents domaines. 


\section{L'éducation, la culture et la stratégie de réduction de la pauvreté}

Il est au moins trois domaines dans lesquels le mouvement maya et l'Accord pour l'identité et les droits des peuples indigènes (AIDPI) ont eu des effets significatifs sur la définition ou l'orientation des politiques publiques au Guatemala. Le premier est celui de l'éducation bilingue et interculturelle avec des propositions fortes pour une réforme générale du système éducatif. Les chiffres sont éloquents : le Guatemala se range parmi les pays d'Amérique latine les plus mal placés en termes de taux d'alphabétisation des adultes et de fréquentation de l'école primaire. Les statistiques du ministère de l'Éducation nationale indiquent que $29,7 \%$ de la population adulte du pays - soit 2,2 millions de gens - étaient analphabètes en 1999. La majeure partie de cette population analphabète réside dans les zones rurales $(77 \%)$, appartient à des groupes indigènes $(61 \%)$ et correspond aux femmes. Des caractéristiques géographiques, ethniques et sexuelles similaires s'appliquent à la population d'âge scolaire qui fréquente l'école primaire. Malgré des progrès sensibles durant les années 1990, le taux réel de fréquentation en 2000 s'établissait autour de $88 \%$. Cette même année, les statistiques du ministère relevaient que 164032 filles âgées de 7 à 12 ans n'allaient pas à l'école. Les régions administratives où s'enregistraient les taux les plus élevés d'analphabétisme et les plus bas de fréquentation scolaire - des taux pour certaines d'entre elles inférieurs à $73 \%$ - étaient les départements du nord, du nordouest et du sud-ouest du pays. Des régions qui connaissent les plus grandes concentrations de population indigène. Ce déficit éducatif, parmi les populations rurales indigènes (touchant notamment les femmes et les jeunes filles), a été souligné par tous les rapports récents concernant la pauvreté et le développement. En moyenne, dans le Guatemala d'aujourd'hui, un indigène a suivi l'école durant 1,6 ans, tandis qu'un non-indigène (rural ou urbain) l'a fréquentée durant 2,4 ans et un non-indigène de la ville 5,4 ans ${ }^{45}$.

De tels écarts montrent l'immensité du défi auquel se trouve confronté le gouvernement du pays pour assurer un égal accès à l'éducation de populations aussi diverses sur le plan ethnique et linguistique, et les premiers pas que le Guatemala a effectués dans le domaine de l'éducation bilingue et interculturelle vont dans ce sens. Depuis les années 1970, en effet, un mouvement très actif, et dont l'importance va croissante, porté par des instituteurs et des linguistes mayas a mis en place des programmes d'éducation bilingue et interculturelle qui, appuyés originellement par des donateurs internationaux, ont été pris en charge depuis par le gouvernement. Au milieu des années 1980, par exemple, le Guatemala a monté le Programme national d'éducation bilingue (PRONEBI) qui proposait une formation spéciale pour les professeurs bilingues et une traduction partielle des programmes scolaires nationaux correspondant aux premières années de l'enseignement primaire dans les quatre principales langues mayas. En 1991, 96194 enfants indigènes, sur un total de 653413 (soit 14,7\% des enfants 
indigènes en âge d'aller à l'école primaire), étaient inscrits dans les écoles du PRONEBI. Dans le même temps, de nombreux instituteurs mayas créaient leurs propres écoles privées destinées aux enfants indigènes, beaucoup d'entre elles panachant l'éducation bilingue et l'apprentissage des valeurs, coutumes et croyances spirituelles mayas. En 1995, après la signature de l'AIDPI, des instituteurs et des linguistes mayas s'étaient regroupés au sein de plusieurs organisations régionales et nationales pour la promotion d'un vaste programme d'éducation bilingue et interculturelle. Ces initiatives conduisirent à la création d'une nouvelle Direction générale pour l'éducation bilingue/interculturelle (DIGEBI) au sein du ministère de l'Éducation nationale. Ces organisations mayas participèrent activement aux débats de la Commission de réforme éducative installée suite aux Accords de paix. Le rapport que rédigea cette dernière soulignait la nécessité d'étendre le programme d'éducation bilingue et interculturelle pour ensuite régionaliser et décentraliser les fonctions du ministère de l'Éducation, de façon à respecter davantage la réalité ethnique et linguistique du pays, et faire entrer l'histoire et les valeurs mayas dans les programmes nationaux. Plus, peut-être, que dans tout autre domaine, les débats et discussions touchant à la réforme de l'éducation ont montré le pouvoir grandissant du mouvement maya, rendant manifeste son influence de plus en plus marquée sur les grandes questions de société et de politiques publiques ${ }^{46}$.

Le deuxième domaine où le mouvement maya et l'AIDPI ont exercé une influence significative est celui de la politique culturelle. Bien que le Guatemala se soit doté d'une Loi du patrimoine culturel dans le milieu des années 1940, ce n'est qu'en 1985, dans le cadre de la nouvelle Constitution nationale, qu'il créa le ministère de la Culture et des Sports. Durant ses premières années, le nouveau ministère exerça des fonctions traditionnelles de conservation du patrimoine archéologique et historique ainsi que d'appui aux activités artistiques, sportives et autres, principalement dirigées vers les populations urbaines et ladinas du pays. Après la signature de l'AIDPI, quelques archéologues travaillant avec l'Institut d'anthropologie et d'histoire (IDAEH, dépendance du ministère de la Culture) se sont impliqués dans un débat des plus sensibles touchant à la participation des indigènes à l'administration et au contrôle de l'héritage archéologique national. Fut soulevée également la question du rôle de l'État dans la protection des lieux sacrés et des sites cérémoniels mayas. En janvier 2000, le nouveau président élu, Alfonso Portillo Cabrera, économiste de formation, créa la surprise en nommant Otilia Lux de Cotí (une éducatrice maya bien connue pour son rôle dans la Commission de la vérité mise en place après les Accords de paix) à la tête du ministère de la Culture et des Sports. Celle-ci, à son tour, nomma un autre leader maya renommé, Virgilio Alvarado Ajanel, comme vice-ministre : tous deux firent savoir publiquement qu'ils allaient consacrer tous leurs efforts à la réforme des politiques culturelles et des structures du ministère en vue de mieux tenir compte des valeurs du multiculturalisme et de l'interculturalisme prônées par les Accords 
de paix. De fait, en mars 2000, deux mois à peine après son entrée en fonction, la nouvelle responsable du ministère annonçait qu'un congrès national de politiques culturelles se tiendrait le mois suivant dans la ville coloniale d'Antigua. Lors de cette annonce, elle déclara à la presse : "Nous vivons dans un pays multilingue, pluriculturel et multi-ethnique [...]. Cette situation, ajoutée au fait que nous sommes au seuil du $\mathrm{xxI}^{\mathrm{e}}$ siècle, nous oblige à chercher et étudier des orientations de politique culturelle dont notre pays a tant besoin $"{ }^{47}$.

Le Congrès national sur les politiques culturelles d'avril 2000 avait pour objectif de réunir quelque 250 personnes identifiées par le ministère comme étant actives dans les domaines de la culture et du développement. Une fois annoncé, ce furent plus de 600 personnes qui s'y inscrivirent, représentant près de 200 organisations et institutions, la plupart d'entre elles professionnelles auxquelles s'ajoutèrent nombre de responsables d'organisations mayas, de représentants des gouvernements locaux (dont des maires et conseillers municipaux des zones rurales). En plus des congressistes mayas, s'y invitèrent une délégation de Garifunas (ou Afro-caribéens) venue de la côte atlantique du Guatemala et un grand nombre de jeunes étudiants du secondaire ou de l'université, venus exprimer les préoccupations d'une partie souvent non prise en compte de la population nationale (dont $44,8 \%$ a moins de 15 ans et $64 \%$ moins de 25 ans). Le congrès fut inauguré par le président Portillo, qui souligna le rôle que la culture et l'identité devaient jouer dans une société et une économie de plus en plus " globales » et déclara que le pays avait un grand besoin de telles propositions de politiques culturelles pour atteindre ses objectifs de démocratisation, de décentralisation et de développement durable. Après la cohorte des discours ofliciels, les participants s'organisèrent en une série d'ateliers et de groupes de travail : aspects législatifs et juridiques de la culture, promotion du développement social et culturel, conservation du patrimoine culturel et naturel, économie des industries et productions culturelles, information et communication pour le développement culturel, les sports et les loisirs... Les recommandations issues de ces réflexions collectives (et parfois animées) furent exposées en session plénière, l'une des plus importantes étant la création d'un comité ad hoc chargé d'accompagner le processus de développement des politiques culturelles, formé de représentants de la société civile, de la communauté académique et du secteur privé, un comité au sein duquel seraient justement représentés les indigènes et les femmes. En novembre 2000 , le ministère de la Culture et des Sports publia les actes du congrès ${ }^{48}$ et émit, sur la base du consensus auquel était parvenu le comité lors de ses travaux ultérieurs, une série de principes et d'objectifs devant guider les actions du gouvernement en matière culturelle. Le principe le plus fondamental posait la reconnaissance, le respect et la promotion de la diversité culturelle et linguistique du Guatemala. De même se voyaient placés au premier plan «la liberté, la participation civique, la solidarité, la responsabilité et l'équité, et le droit pour quiconque de participer à la vie culturelle et interculturelle du pays ». L'affirma- 
tion d'une telle politique culturelle se veut profondément respectueuse des valeurs culturelles, des cosmologies, des modes de comportements, des croyances et pratiques des divers peuples qui composent le Guatemala, et se pose comme une donnée incontournable dans la lutte pour le développement humain. « Pour cette raison, l'État est obligé de tenir compte de la dimension culturelle dans tous ses plans et actions visant à améliorer la qualité de vie et la capacité de réalisation personnelle de chacun des habitants de ce pays $\gg{ }^{49}$.

La nouvelle politique culturelle promue par le ministère de la Culture et des Sports s'attaque aussi à la nécessaire projection de la dimension culturelle dans tous les programmes gouvernementaux et à tous les niveaux, départemental, municipal, communautaire. Cela implique l'intégration de promoteurs culturels au sein de " conseils de développement » départementaux. D’autres points sont mis en avant, tels que la nécessité de promouvoir la révision et l'actualisation de la législation culturelle, la formation de professionnels et l'appui à la recherche dans le domaine culturel, mais aussi et surtout le renforcement institutionnel du ministère de la Culture et la collaboration avec d'autres instances gouvernementales ou non gouvernementales soucieuses de promouvoir le développement ${ }^{50}$.

Le troisième et dernier domaine dans lequel le mouvement maya et l'AIDPI ont exercé une influence (quoique encore limitée) est celui de la formulation de la stratégie de réduction de la pauvreté (ERP). La responsabilité de la préparation de ce programme national revint au Secrétariat au plan (SEGEPLAN, dépendant de la Présidence de la république) avec l'appui des agences internationales bailleuses de fonds et de la communauté universitaire. L'essentiel du travail de SEGEPLAN consista à reprendre, actualiser et étendre la base de données en tenant compte de tous les indicateurs de pauvreté et de développement humain au Guatemala. Un premier et court rapport fut publié en février 2001, suivi par la publication de la Carte de la pauvreté au Guatemala qui montrait la situation des différentes régions du pays et identifiait 102 bourgades où la situation était la plus critique. Ainsi que l'avaient déjà souligné plusieurs rapports antérieurs (produits par le gouvernement ou les agences internationales), la pauvreté - et souvent l'extrême pauvreté - et le manque d'accès aux services sociaux de base sont en corrélation étroite avec l'habitat rural et correspondent aux régions géographiques qui connaissent les plus hautes concentrations de populations indigènes ${ }^{51}$.

Étant donnée l'attention particulière que le ministère de la Culture et des Sports portait à la dimension culturelle du développement, spécialement dans le contexte de la reconnaissance de la richesse multiculturelle de la société guatémaltèque, celui-ci ouvrit des discussions avec SEGEPLAN afin qu'il introduise pleinement cette dimension dans ses travaux sur l'ERP. Une réunion de travail sur le thème "Culture et pauvreté », organisée à la capitale par le ministère de la Culture, SEGEPLAN, la Faculté latino-américaine de Sciences sociales (FLACSO) et l'Institut de recherches économiques et sociales (IEDES) de l'université Rafael Landívar, qui regroupait des spécialistes nationaux et internatio- 
naux, fut une première : comment une politique culturelle explicite, telle que le ministère l'imposait pour respecter la diversité et le pluralisme, peut-elle cadrer avec une stratégie visant à réduire la pauvreté et à promouvoir le développement durable? Un texte préliminaire préparé par le ministère proposait trois projets ayant trait aux instituts de formation aux langues régionales, à la promotion de la production artisanale et d'autres industries culturelles, et à la co-gestion de parcs et autres aires protégées par des communautés indigènes. Durant la réunion, plusieurs communications donnèrent une place privilégiée au rôle de la culture dans les interventions de développement (santé, éducation, etc.), dans la gestion de l'environnement et de la décentralisation, et dans la participation sociale et politique. " Le séminaire, selon le document final, a ouvert un espace au dialogue multiculturel, multisectoriel et multidisciplinaire concernant des thèmes aussi fondamentaux pour le développement durable au Guatemala que sont la pauvreté, la marginalisation culturelle, l'identité nationale, la potentialité économique des ressources culturelles, la conservation de l'environnement, la participation sociale et montré les voies que le multiculturalisme et la compréhension interculturelle pouvaient ouvrir dans la stratégie de réduction de la pauvreté » ${ }^{52}$.

Depuis cette réunion de juin 2001, SEGEPLAN a proposé une ébauche de ce que devrait prendre en compte sa stratégie de réduction de la pauvreté. L'objectif fondamental est de promouvoir l'équité dans l'orientation des dépenses publiques en assignant un plus fort pourcentage de ressources aux programmes et projets destinés aux populations les plus pauvres du pays, et notamment aux populations rurales et indigènes. Entre autres choses, cela appelle une modernisation du système actuel de gestion de l'argent public, une plus grande transparence dans l'utilisation de ces fonds, une participation croissante des citoyens et une décentralisation des programmes de réduction de la pauvreté vers les niveaux municipal et communautaire. Stratégiquement, l'ERP suppose une modernisation du système financier et une stabilité macro-économique, de plus gros investissements dans le capital humain (notamment dans la santé et l'éducation), ainsi que dans les infrastructures (construction et entretien des routes et chemins ruraux, électrification et salubrité de l'eau). Pour chacune de ces activités, l'ERP prévoit une série de dispositions visant à respecter la diversité culturelle, la compréhension et le dialogue interculturel, la qualité de l'environnement, l'équité sexuelle et l'amélioration de la gestion des risques naturels ${ }^{53}$.

La situation politique et économique du Guatemala est telle qu'il est diffícile de savoir où en est l'actuel gouvernement ${ }^{54}$ quant au programme de réduction de la pauvreté tel qu'il est dessiné dans le document de l'ERP. Le mener à bien demanderait en effet un accroissement significatif des ressources publiques dédiées à la réduction de la pauvreté, un usage plus efficace et transparent de ces ressources, une plus grande ouverture à la participation citoyenne et une décentralisation des budgets et des initiatives. Autant de points qui sont loin d'être acquis dans le cadre des conceptions et des pratiques traditionnelles de la politi- 
que au Guatemala. Cependant, malgré ces contraintes (qui sont bien réelles et dénoncées quotidiennement par la presse nationale), le Guatemala est l'un des premiers pays d'Amérique latine à tenir explicitement compte d'une dimension culturelle dans la stratégie qu'il propose pour réduire la pauvreté. D'autres pays latino-américains, tels que la Bolivie, l'Équateur, le Mexique ou le Pérou, où se sont développées de fortes organisations indigènes, auraient également les capacités constitutionnelles et législatives pour introduire cette dimension multi- et interculturelle dans leurs programmes nationaux de développement. Comme au Guatemala, tout dépendra dans ces pays de la volonté politique des gouvernants, des partis et des élites qui exercent depuis si longtemps le contrôle du pouvoir. Toutefois, quelle que soit la direction que suive dans l'avenir la politique guatémaltèque, il semble qu'elle ne pourra pas ignorer les avancées obtenues par le mouvement maya et les accords concernant les peuples indigènes. Le Guatemala, comme ces autres pays du continent à fortes populations indigènes, est face à un défi immense : créer une future nation dans laquelle serait enfin respectée la diver sité culturelle et linguistique, où règneraient l'acceptation, la compréhension et la communication mutuelles entre des peuples partageant différentes cultures et histoires ethniques, et où la pauvreté, l'inégalité et l'exclusion seraient combattues et réduites. La question est de savoir si et comment un tel objectif peut être atteint au plus vite en profitant des (encore) fragiles structures démocratiques au sein desquelles les peuples indigènes et leurs organisations ont conquis et gagné une participation active, sans retomber dans le « nationalisme exclusif » et plonger de nouveau dans les violences politiques du passé.

Shelton H. DAvis, Banque mondiale

Traduit et adapté de l'américain par Alain Breton

\section{NOTES}

* Cet article est repris de « The Mayan movement and national culture in Guatemala », publié dans Vijayendra Rao et Michael Walton, éds, Culture and public action, Stanford University Press, Stanford, 2004, pp. 328-358, avec l'aimable autorisation de l'auteur et des éditeurs (C International Bank for Reconstruction and Development/World Bank).

1. Voir "Introducción ", in Guillermo Bonfil Batalla, éd., 1981, Utopía y revolución : el pensamiento político contemporáneo de los Indios en América Latina (Editora Nueva Imagen, Mexico).

2. Voir Donna Lee Van Cott, éd., 1994, Indigenous peoples and democracy in Latin America (Saint Martin's Press, New York).

3. Voir, entre autres, Alan Le Baron, 1993, "The creation of the modern Maya ", in Crawford Young, éd., The rising tide of cultural pluralism : the nation state at bay ? (University of Visconsin Press, Madison); Edward Fischer et R. McKenna Brown, éds, 1996, Mayan cultural activism in Guatemala (University of Texas Press, Austin); Kay B. Warren, 1998, Indigenous movements and their critics : Pan-Maya activism in Guatemala (Princeton University Press, Princeton). 
4. La littérature ethnographique et sociologique concernant les Mayas et les autres peuples indigènes du Guatemala est vaste et trop abondante pour être entièrement citée ici. Cependant, pour une vue d'ensemble de la situation actuelle des peuples indigènes du Guatemala, on se reportera au rapport publié par l'Unité régionale d'assistance technique (RUTA) de la Banque mondiale et à l'étude du ministère de la Culture et des Sports du Guatemala, Perfil de los pueblos Maya, Garifina y Xinka de Guatemala (2001).

5. Concernant les niveaux de pauvreté et de développement au Guatemala, on se reportera au rapport du Programme des Nations unies pour le développement (PNUD), Guatemala : la fuerza incluyente del desarrollo humano (Naciones unidas, Guatemala, 2000). Voir aussi El Drama de la pobreza en Guatemala : sus rasgos y efectos sobre la sociedad (Gobierno de la República de Guatemala, Guatemala, février 2001).

6. Sur l'histoire de l'économie du café au Guatemala et sa relation avec l'économie indigène des hautes terres ou Altiplano, voir Julio Castellanos Cambranes, 1985, Coffee and peasants : the origins of the modern plantation economy in Guatemala, 1853-1897 (Institute of Latin American Studies, Stockholm) et David McCreery, 1994, Rural Guatemala, 1760-1940 (Stanford University Press, Stanford).

7. Sur la nature exclusive de l'État guatémaltèque et son irrespect total des droits et des cultures mayas, voir Carol A. Smith, éd., 1990, Guatemalan Indians and the state : 1540 to 1988 (University of Texas Press, Austin) et Arturo Taracena Arriola, 1999, "Proyecto nacional y nación multicultural », in Jorge Nieto Montesinos, éd., Sociedades multiculturales y democracia en América Latina (UNESCO, Mexico), pp. 261-272.

8. Voir Edelberto Torres Rivas, 1971, Interpretación del desarrollo social centroamericano : procesos y estructuras de una sociedad dependiente (Editorial Universitaria Centroamericana, San José, Costa Rica), chap. 2. L'essentiel de la présentation historique qui suit est repris de Shelton H. Davis, 1988, "Agrarian structure and ethnic resistance: the Indian in Guatemalan and Salvadoran national politics ", in Remo Guidieri, Francisco Pellizzi et Stanley J. Tambiah, éds, Ethmicities and nations : processes of interethmic relations in Latin America, Southeast Asia, and the Pacific (University of Texas Press, Austin), pp. 78-106.

9. L'historique du développement de la production de café et de ses effets sur les communautés indigènes peut être consulté dans Sanford A. Mosk, 1955, « The coffee economy of Guatemala, 1850-1918. Development and signs of instability ", Inter-American Economic Affairs, 9 (3), pp. 6-20, et dans les ouvrages précédemment cités de Castellanos Cambranes (op. cit., 1985) et McCreery (op. cit., 1994).

10. Voir Shelton H. Davis, 1970, « Land of our ancestors : a study of land tenure and inheritance in the Highlands of Guatemala » (Ph.D. dissertation, Harvard University). Ce travail a été publié en 1997, en espagnol sous le titre La Tierra de nuestros antepasados : estudio de la herencia y la tenencia de la tierra en el Altiplano de Guatemala (Centro de Investigaciones Regionales de Mesoamérica/Plumsock Mesoamerican Studies, Antigua, Guatemala).

11. Antonio Batres Jaúregui, 1894, Los Indios, su historia y su civilización (Establecimiento Tipográfico La Unión, Guatemala), pp. 160-162.

12. Antonio Batres Jaúregui, op. cit., p. 172.

13. Antonio Batres Jaúregui, op. cit., p. 159.

14. Antonio Batres Jaúregui, op. cit., pp. 177-178. Cette citation est reprise du livre de Greg Grandin, 2000, The Blood of Guatemala : a history of race and nation (Duke University Press, Durham), p. 142 et note 40 , p. 284. Cet ouvrage propose une histoire des relations interethniques à Quetzaltenango, la deuxième ville du Guatemala qui fut le centre d'un mouvement de résurgence maya à la fin du $\mathrm{XIX}^{\mathrm{e}}$ siècle.

15. Pour un exposé plus détaillé de l'histoire de cette notion discriminatoire de citoyenneté au Guatemala, voir Taracena Arriola (op. cit., 1999) et la section historique du rapport du PNUD citée en note 5 .

16. Parmi les recherches ethnographiques portant sur les communautés mayas des hautes terres durant cette période, voir Oliver La Farge, 1947, Santa Eulalia : the religion of a Cuchumatan Indian town (University of Chicago Press, Chicago) ; Ruth Bunzel, 1952, Chichicastenango : a Guatemalan 
village (University of Washington Press, Seattle) ; Franz Termer, 1957, Etnologia y etnografia de Guatemala (Seminario de Integración Social Guatemalteca, Guatemala). Pour une discussion plus générale touchant à la persistance de la culture et de l'identité mayas au niveau local, voir Sol Tax, 1937, « The Municipios of the Midwestern Highlands of Guatemala ", American Anthropologist, 39, pp. 413-444.

17. Sol Tax, 1941, "World View and Social Relations in Guatemala ", American Anthropologist, 43 , pp. $27-42$.

18. Cette interprétation rejoint celle que proposait Éric Wolf concernant l'impact du colonialisme européen et la nouvelle économie politique mondiale sur les peuples indigènes, notamment dans l'ouvrage de 1982, Europe and the people without history (University of California Press, Berkeley).

19. Pour une étude détaillée de cette période - et des réactions du gouvernement des États-Unis face aux réformes sociales menées par les gouvernements d'Arévalo et d'Árbenz -, voir Piero Gleijeses, 1991, Shattered hope : the Guatemalan revolution and the United States, 1944-1954 (Princeton University Press, Princeton).

20. Sur les changements politiques et législatifs qui ont marqué cette période, en particulier ceux qui se rapportent à la population indigène rurale, voir Jorge Skinner-Klee, 1954, Legislación indigenista de Guatemala (Instituto Indigenista Interamericano, Mexico) et Kalman H. Silvert, 1954, A study in government : Guatemala (Tulane University, New Orleans, coll. « Middle American Research Institute Publication » 21).

21. Pour une synthèse de quelques-uns des résultats de ces études, voir l'article du fondateur et premier directeur de l'INI, Antonio Goubaud Carrera, 1959, " Adaptación del Indígena a la cultura nacional moderna ", in Cultura indigena de Guatemala : ensayos de antropología social (Seminario de Integración Social Guatemalteca, Guatemala).

22. Ces chiffres sont repris de James Handy, 1994, Revolution in the countryside : rural conflict and agrarian reform in Guatemala, 1944-1954 (University of North Carolina Press, Chapel Hill), pp. 13, 82.

23. Le contenu de ces enquêtes sur les terres municipales et communales est traité dans José Luis Paredes Moreira, 1963, Reforma agraria : una experiencia en Guatemala (Imprenta Universitaria, Guatemala), pp. 101-121.

24. On trouvera des détails concernant le contenu de la loi de réforme agraire dans les travaux précédemment cités de Paredes Moreira (1963), Gleijeses (1991) et Handy (1994). L'étude de la Banque mondiale dont il est question dans ce paragraphe a été publiée sous forme d'un livre sous le titre The economic development of Guatemala (International Bank for Reconstruction and Development, Washington, 1951) et résumée par le chef de mission de la Banque mondiale G. E. Britnell, 1951, dans un article intitule " Problems of economic and social change in Guatemala », The Canadian Journal of Economics and Political Science, 17 (4), pp. 468-481.

25. Le rôle des politiciens locaux, notamment dans leur relation aux conflits sur les terres municipales durant la Réforme agraire, est traité dans Handy (op. cit., 1994). Voir aussi les articles de John Gillin et Kalman H. Silvert, 1956, " Ambiguities in Guatemala », Foreign Affairs, 34 (3), pp. 469-482, et Robert Wasserstrom, 1975, «Revolution in Guatemala : peasants and politics under the Árbenz government ", Comparative Studies in Society and History, 17 (4), pp. 443-478.

26. Pour une analyse et une illustration des effets de l'accroissement démographique sur la tenure et l'usage des terres, on reportera le lecteur à Shelton H. Davis (op. cit., 1997), pp. 127-156.

27. On lira une description de cette mobilisation politique au sein de plusieurs centres communautaires indiens dans Richard N. Adams, éd., 1957, Political changes in Guatemalan Indian communities (Tulane University, New Orleans, 1957, coll. « Middle American Research Institute Publication » 21).

28. Pour une compréhension de l'amplification et de l'influence de l'Église catholique romaine dans les années post-Árbenz, voir Phillip Berryman, 1984, The Religious roots of rebellion : christians in central american revolutions (Orbis Books, New York). Il existe plusieurs études sur le rôle du mouvement d'Action catholique tel qu'il s'est développé dans les communautés indiennes: on lira notamment Kay B. Warren, 1978, The symbolism of subordination : Indian identity in a Guatemalan town (University of Texas Press, Austin) et Ricardo Falla, 1978, Quiche rebelde (Editora Universitaria, Guatemala). 
29. La région où l'Action catholique s'établit avec le plus de vigueur et où l'idéologie était la plus marquée fut le département du Quiché, là même où les prêtres du Sacré-Câur de Jésus avaient été actifs dès la fin des années 1950. Tout au long des années 1960 et 1970, les missionnaires du Sacré-Cœur non seulement convertirent au nouveau catholicisme un très grand nombre d'Indiens, mais mirent sur pied de nombreuses ligues paysannes, des coopératives agricoles et des programmes de colonisation de terres. Il n'est pas surprenant que cette région, et notamment l'aire ixil du Nord-Quiché, soit celle où s'est implantée et développée l'organisation de la guérilla parmi les Mayas. Pour plus de détails, voir L. Fried, Marvin E. Gettleman, Deborah T. Levenson et Nancy Peckenham, éds, 1983, Guatemala in rebellion : unfinished history (Grove Press, New York) et Yvon Le Bot, 1992, La guerre en terre maya. Communauté, violence et modernité au Guatemala, 1970-1992 (Karthala, Paris).

30. La première tentative pour former un parti politique indigène remonte à cette période, mais ses quelques leaders mayas furent rapidement récupérés par les partis traditionnels. Pour plus de détails, voir Ricardo Falla, 1978, "El Movimiento indigena », Estudios Centroamericanos, 33 (356-357), pp. 437-461.

31. La renaissance du mouvement syndical guatémaltèque est amplement analysée par Roger Plant, 1978, Guatemala : unnatural disaster (Latin America Bureau, Londres).

32. L'action du CUC dans le ralliement des travailleurs agricoles et des paysans indiens, comme dans le développement de la guérilla dans les zones majoritairement indigènes du pays, est décrite et analysée dans Jim Handy, 1984, Gift of the devil : a history of Guatemala (South End Press, Boston).

33. Pour ce qui concerne les origines sociales et les conséquences du mouvement de guérilla comme des campagnes anti-insurrectionnelles qui ont ravagé le Guatemala entre la fin des années 1970 et le début des années 1980, voir Robert M. Carmack, éd., 1988, Harvest of violence : the Maya Indians and the Guatemala crisis (University of Oklahoma Press, Norman) et Le Bot (op. cit., 1992). Depuis la publication de ces ouvrages, écrits par des scientifiques ayant travaillé sur le terrain dès avant la violence, de nombreuses études ont traité du sujet, entre autres le rapport de la Commission de clarification historique (CEH), 1999, Guatemala : memory of silence (CEH, Guatemala).

34. On rappellera que Guillermo Bonfil Batalla fut l'un des premiers observateurs étrangers à identifier les balbutiements d'un mouvement maya moderne au Guatemala. Il y a un quart de siècle, dans son livre, Utopía y Revolución... (voir note 1), il reproduisait déjà deux articles écrits par des Mayas, le premier par l'intellectuel et juriste q'eqchi' Antonio Pop Caal et le second par un des rédacteurs d'Ixim, le premier périodique en langue maya du Guatemala. Pour des travaux récents sur l'origine, l'évolution et les différents courants qui traversent le mouvement maya, on se reportera à Edward Fischer et R. McKenna Brown, éds, 1996, Mayan cultural activism in Guatemala (University of Texas Press, Austin), ainsi qu'à Kay B. Warren, 1998, Indigenous movements and their critics : Pan Maya activism in Guatemala (Princeton University Press, Princeton).

35. La biographie de Rigoberta Menchú, 1983, Moi, Rigoberta Menchú. Une vie et ume voix, la révolution au Guatemala (Gallimard, Paris), décrit la vie des Mayas ainsi que la montée en puissance de la violence et les atrocités de la répression militaire lors des campagnes anti-insurrectionnelles menées contre sa famille et d'autres villageois mayas. Le livre fut écrit en collaboration avec l'anthropologue française Elisabeth Burgos-Debray; il a beaucoup contribué à faire connaitre la condition des indigènes et la situation des droits de l'homme au Guatemala auprès de l'opinion internationale et, sans doute, joué un rôle non négligeable dans l'attribution du prix Nobel de la paix à Rigoberta Menchú.

36. Sur cette hétérogénéité au sein du mouvement maya, voir Santiago Bastos et Manuela Camus, 1993, Quebrando el silencio : organizaciones del pueblo Maya y sus demandas (1986-1992) (FLACSO, Guatemala) et Alberto Esquit Choy et Víctor Gálvez Borell, 1997, The Mayan Movement Today': Issues of Indigenous Culture and Development in Guatemala (FLACSO Guatemala).

37. Certaines de ces craintes furent exprimées dans les écrits de Mario Roberto Morales, un intellectuel guatémaltèque jadis lié à la Gauche qui, à son retour d'exil, commença à critiquer régulièrement ce qu'il considérait comme des contradictions inhérentes au mouvement maya. Voir, par exemple, son article, "Esencialismo "Maya", mestizaje Ladino y nación inter-cultural : los discursos 
en debate », in Clara Arenas Bianchi, Charles R. Hale et Gustavo Palma Murga, éds, 1999 ¿ Racismo en Guatemala? Abriendo el debate sobre un tema tabú (AVANCSO, Guatemala), pp. 217-272.

38. Voir Jorge Dandler, 1999, «Indigenous peoples and the rule of law in Latin America : do they have a chance? ", in Juan E. Méndez, Guillermo O'Donnell et Paulo Sergio Pinheiro, éds, The (Un) rule of law and the underprivileged in Latin America (University of Notre Dame Press, Notre Dame), pp. 116-151.

39. Sur les débats soulevés par la ratification de la Convention 169 de l'OIT, voir Bastos et Camus, op. cit., 1993, et Suzanne Jonas, 2000, Of Centaurs and doves : Guatemala's peace process (Westview Press, Boulder).

40. Voir Acuerdo sobre identidad y derechos de los pueblos indigenas (Presidencia de la República, Comisión de la Paz, Guatemala, 1996) et Saqb'ichil COPMAGUA, Acciones Politicas y Organizativas (Saqb'ichil COPMAGUA, Guatemala, 2000).

41. Pour plus de détails, on se reportera aux travaux de la commission nationale permanente de réforme éducative, Proyecto de reforma educativa desde la perspectiva indigena (Saqb'ichil COPMAGUA, Guatemala, 1998) et de la Commission paritaire de réforme éducative, Diseño de reforma educativa : Runuk'ik Jun K'ak'a Tijonik (Proyecto de Interculturalidad, Políticas Públicas y Desarrollo Humano Sostenible, Q'anil B, Guatemala, 1998).

42. Le thème de la reconnaissance par l'État des procédures et des lois coutumières indigènes a été l'un de ceux qui ont tenu une place centrale lors des débats qui ont accompagné et prolongé la ratification de la Convention 169 de l'OIT et l'application de l'AIDPI. Voir, entre autres, Flavio Rojas Lima, 1995, El Derecho consuetudinario en el contexto de la etmicidad guatemalteca (Procuraduria de los Derechos Humanos, Guatemala).

43. Voir Shelton H. Davis, 1997, "The Guatemalan peace accords and indigenous communal lands » (communication présentée au Colloquium Series of the Program in Agrarian Studies, Yale University).

44. Suzanne Jonas, 2000, Of Centaurs and doves: Guatemala's peace process (Westview Press, Boulder), chap. 8 .

45. Chiffres calculés à partir des rapports du ministère de l'Éducation nationale et de l'étude publiée par le Gouvernement de la république de Guatemala, El Drama de la pobreza en Guatemala : sus rasgos y' efectos sobre la sociedad (février 2001, Guatemala).

46. Voir Julia Becker Richards et Michael Richards, 1996, « Mayan education : an historical and contemporary analysis of mayan language education policy », in Edward Fischer et R. McKenna Brown, éds, op. cit., pp. 208-221 ; World Bank, 1995, Guatemala : basic education strategy. equity and efficiency in education (Human Resources Operations Division, Country Department II, Latin America and Caribbean Regional Oflice, Report $\mathrm{n}^{\circ}$ 12204-GU, Washington) et Comisión paritaria de reforma educativa, 1998, Diseño de reforma educativa : Rumuk'ik Jun K'ak'a Tijonik (Guatemala).

47. Cité dans Miriam de León, 2000, « Políticas culturales sobre el tapete », Prensa Libre, 16 mars.

48. Ministère de la Culture et des Sports, 2000, Informe final : congreso nacional sobre lineamientos de politicas culturales (11-14 April 2000) (novembre 2000, Guatemala).

49. Ministère de la Culture et des Sports, 2000, Políticas culturales y deportes nacionales (Guatemala).

50. Ibid., p. 4.

51. Voir SEGEPLAN, 2001, El drama de la pobreza en Guatemala : un informe sobre los rasgos de esta orivación y sus efectos sobre la sociedad (février 2001, Guatemala) et Mapas de pobreza de Guatemala : instrumentos para entender el flagelo de la pobreza en el pais (août 2001, Guatemala).

52. Ministère de la Culture et des Sports/SEGEPLAN, Seminario-Taller : cultura y pobreza. Informe final (juin 2001, Guatemala).

53. SEGEPLAN, Estrategia de reducción de la pobreza : un camino para la paz (propuesta para discusión) (13 septembre 2001, Guatemala, p. 39).

54. Formé par le nouveau président de la République, Oscar Berger (du parti libéral GANA, Gran Alianza Nacional), élu en décembre 2003 et entré en fonction en janvier 2004 (NdT). 